\title{
High-gradient testing of an $S$-band, normal-conducting low phase velocity accelerating structure
}

\author{
A. Vnuchenko๑, ${ }^{*}$ D. Esperante Pereira, and B. Gimeno Martinez \\ Instituto de Fsica Corpuscular (IFIC), 46980 Valencia, Spain
}

S. Benedetti®, N. Catalan Lasheras, M. Garlasch๑, A. Grudiev, G. McMonagle, S. Pitman, I. Syratchev $\odot$, M. Timmins, R. Wegner॰, B. Woolley, and W. Wuensch $\odot$

CERN, European Organization for Nuclear Research, 1211 Geneva, Switzerland

A. Faus Golfe

Laboratory of the Physics of the two infinities Irène Joliot-Curie (IJCLab), CNRS/IN2P3, 91898 Orsay, France

(Received 5 January 2020; accepted 29 July 2020; published 31 August 2020)

\begin{abstract}
A novel high-gradient accelerating structure with low phase velocity, $v / c=0.38$, has been designed, manufactured and high-power tested. The structure was designed and built using the methodology and technology developed for CLIC $100 \mathrm{MV} / \mathrm{m}$ high-gradient accelerating structures, which have speed of light phase velocity, but adapts them to a structure for nonrelativistic particles. The parameters of the structure were optimized for the compact proton therapy linac project, and specifically to $76 \mathrm{MeV}$ energy protons, but the type of structure opens more generally the possibility of compact low phase velocity linacs. The structure operates in S-band, is backward traveling wave (BTW) with a phase advance of 150 degrees and has an active length of $19 \mathrm{~cm}$. The main objective for designing and testing this structure was to demonstrate that low velocity particles, in particular protons, can be accelerated with high gradients. In addition, the performance of this structure compared to other type of structures provides insights into the factors that limit high gradient operation. The structure was conditioned successfully to high gradient using the same protocol as for CLIC X-band structures. However, after the high power test, data analysis realized that the structure had been installed backwards, that is, the input power had been fed into what is nominally the output end of the structure. This resulted in higher peak fields at the power feed end and a steeply decreasing field profile along the structure, rather than the intended near constant field and gradient profile. A local accelerating gradient of $81 \mathrm{MV} / \mathrm{m}$ near the input end was achieved at a pulse length of $1.2 \mu \mathrm{s}$ and with a breakdown rate (BDR) of $7.2 \times 10^{-7} 1 / \mathrm{pulse} / \mathrm{m}$. The reverse configuration was accidental but the operating with this field condition gave very important insights into high-gradient behaviour and a comprehensive analysis has been carried out. A particular attention was paid to the characterization of the distribution of BD positions along the structure and within a cell.
\end{abstract}

DOI: 10.1103/PhysRevAccelBeams.23.084801

\section{INTRODUCTION}

A major effort is being made by the CLIC collaboration to develop accelerating structures capable of accelerating relativistic leptons with a gradient in excess of $100 \mathrm{MV} / \mathrm{m}$. A set of design, fabrication and operational procedures have been established and over twenty prototype X-band structures have operated in dedicated test stands above

\footnotetext{
*anna.vnuchenko@cern.ch, anna.vnuchenko@ific.uv.es
}

Published by the American Physical Society under the terms of the Creative Commons Attribution 4.0 International license. Further distribution of this work must maintain attribution to the author(s) and the published article's title, journal citation, and DOI.
$100 \mathrm{MV} / \mathrm{m}$ for extended periods [1-5]. The success of this program opens the opportunity of using high-gradients in other applications. The peak surface field in a structure is often used to characterize the performance of a structure, but more recently it has been shown that other parameters, such as power flow and group velocity, also determine the ultimate structure performance.

One potential application of the high-gradient technology is proton therapy based on linacs. Linacs are an alternative to the synchrocyclotron-based facilities which currently dominate the commercial market. Linac-based proton facilities can offer greater flexibility in treatment parameters and possibly lower cost. A linac-based facility is being developed by ADAM [6,7]. Higher gradient versions of such a concept have been under study by the TERA 
foundation and a collaboration with CLIC on the highgradient structures and high-power rf systems have been underway for some years $[8,9]$. In order to advance this application further, a program was established, and funded by the CERN Knowledge Transfer (KT) fund, to design, build and test a prototype high-gradient structure for proton acceleration, in particular for use in TULIP (turning linac for protontherapy) [10]. This structure is the subject of this report.

The structures would be used to generate a $76 \mathrm{MeV}$ beam, which is the lowest energy planned for the linacmounted gantry of the TULIP facility [10]. Initial studies indicated that high-gradient becomes increasingly difficult to maintain as phase velocity, which must match particle velocity, is reduced below $c$ [11]. The specific target of $v / c=0.38$ was chosen because it is with the least phase velocity making the most challenging structure and it is worth studying whether it is still capable of achieving a gradient of at least $50 \mathrm{MV} / \mathrm{m}$. This is over a factor of two higher than the gradient in the ADAM linac for example. The result of the optimization is a backward traveling wave S-band structure with magnetic cell-to-cell coupling, which is quite different than the forward travelling wave, X-band, speed of light phase velocity CLIC structures. The significant difference in rf parameters compared to CLIC structures makes the BTW structure a very important test for the range of applicability of the CLIC high-gradient design methodology.

This report describes the full development of the prototype: rf design, mechanical design, fabrication, and highgradient testing. The report first describes the structure as designed and operating in the normal configuration. The high-gradient test happened to take place in a reversed power flow direction so that the test result was analyzed based on it. The implications and consequences of the reversed installation are addressed where relevant, and high-gradient results are then compared to CLIC prototype structures.

\section{RADIO FREQUENCY DESIGN AND OPTIMIZATION}

The rf design and optimization of the high-gradient low phase velocity BTW structure is described in detail in Refs. [10-13]. In this report, a short summary is given to highlight the novel features compared to standard S-band side coupled standing wave structures studied previously $[14,15]$. The cell features a shape of the central part of the iris, the so-called nose cone, with both reduced modified pointing vector $S_{c}$, described in [5], and rf power required for a given gradient, optimized using the quantity:

$$
\mu=\frac{P_{z}}{E_{a}^{2}} \cdot \frac{S_{c}}{E_{a}^{2}}=\frac{v_{g}}{\omega} \cdot \frac{S_{c} / E_{a}^{2}}{R^{\prime} / Q}
$$

where $P_{z}$ is the input power in a cell, $E_{a}$ is the cell accelerating gradient, $v_{g}$ is the group velocity in the cell, $\omega$ is the angular $\mathrm{rf}$ frequency, $R^{\prime}$ is the effective shunt impedance per unit length and $Q$ is the quality factor of the cell. Equation (1) weighs equally the required power and the modified Poynting vector. Minimizing $\mu$ is expected to give the highest sustainable gradient for the lowest input power.

The geometry of a full disk of one regular cell is shown in Fig. 1 (top). The shape of a regular (i.e., not coupling) cell is shown together with the variation of the surface electric and magnetic fields as well as the variation of $S_{c}$ in Fig. 1 (bottom).

The cell-to-cell coupling is provided by 16 holes in the region of the highest magnetic field. This gives a magnetic coupling and results in a backward travelling wave, that is a negative phase velocity, and thus the direction of particle acceleration is in the direction opposite to the power flow. The number and size of the coupling holes has been optimized in order to provide the required coupling while limiting the $S_{c}$ values in the region of the coupling hole to a value below that on the nose cone. The variation of $S_{c}$ in the regular cell section is shown in Fig. 1 (bottom right), where the two highest values of $S_{c}$, on the nose cone and in the coupling holes, are roughly equal.

The design is approximately constant-gradient. The group velocity ranges from $0.4 \%$ to $0.2 \%$ of $c$. The range has been chosen as a compromise between acceptable filling time and efficient acceleration of the beam. The group velocity is tapered by varying the radii of the coupling holes. The radial position of the coupling holes has been varied to adjust the resonant frequency. All the other geometrical parameters have been kept constant throughout the structure.
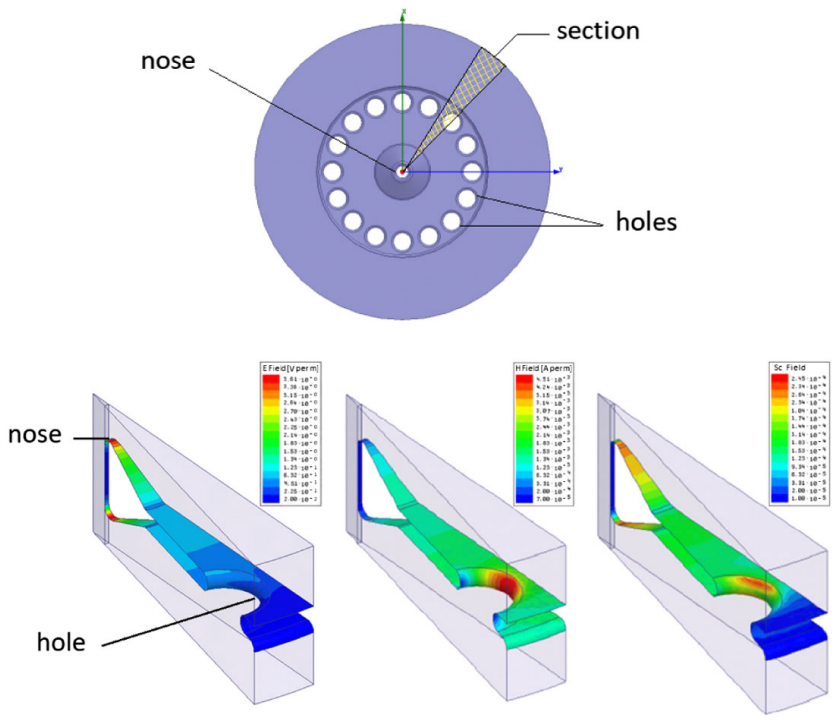

FIG. 1. Regular disk shape (top). Normalized electric field $E / E_{a}$ (left), normalized magnetic field $H / E_{a}$ (center), normalized modified Poynting vector $S_{c} / E_{a}^{2}$ (right) in a regular cell section (1/32 azimuthal symmetry) (bottom). 

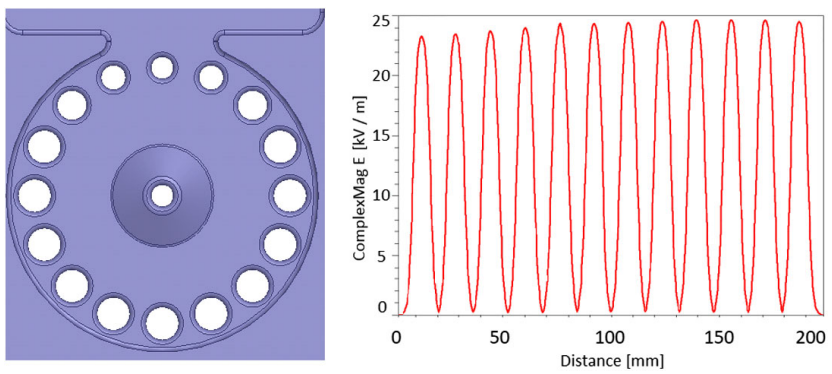

FIG. 2. Input coupling cell (left) and electric field profile in the structure (right). Note the variation in the coupling hole radii.

An analysis of the frequency sensitivity to dimensional changes has been made for the final shape of the regular cell to determine mechanical tolerances and establish the tuning methodology. The tolerances specified in the mechanical drawings result in no more than $\pm 5 \mathrm{MHz}$ of frequency shift per cell. This possible error can be compensated by means of 4 dimple tuners. From simulations and mechanical tests on copper cells, the tuning range is higher than $6 \mathrm{MHz}$ per cell, well beyond the range required in the worst case tolerance scenario.

The input and output power couplers are single-feed with magnetic coupling. They have been optimized to provide impedance matching while perturbing as little as possible the field distribution and the accelerating voltage of the coupling cell. The radius of the coupling holes in the input coupling cell was reduced in the region near the coupling slot in order to compensate for the enhancement of $S_{c}$ due to the local increase of the power flow. The remaining coupling holes were resized to maintain the overall cell-tocell coupling in the cell at the level of the regular cell

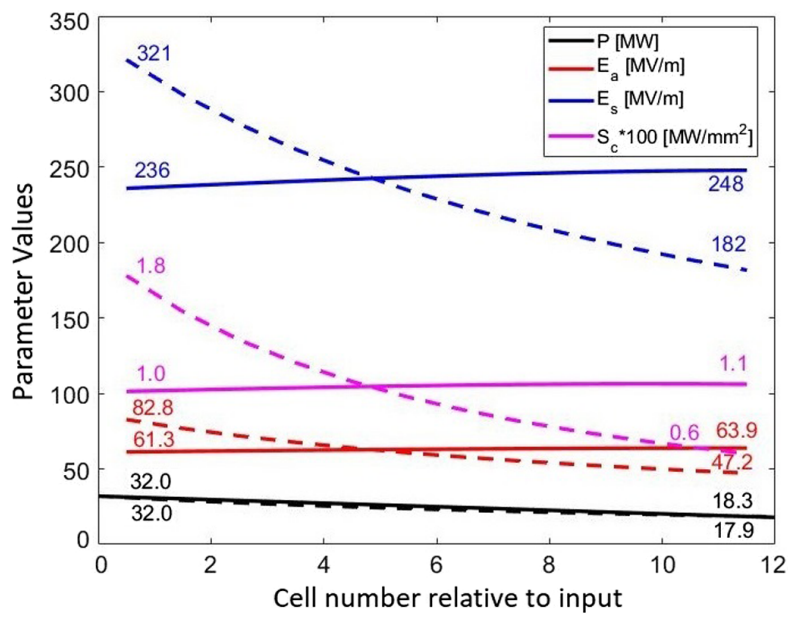

FIG. 3. Variation of the rf parameters along the structure cells at normal (solid) and backward (dash) filling with $32 \mathrm{MW}$ input power; red: accelerating gradient, $E_{a}$; blue: maximum surface electric field, $E_{s}$; magenta: maximum modified Poynting vector, $S_{c}$; black: input power, $P$.
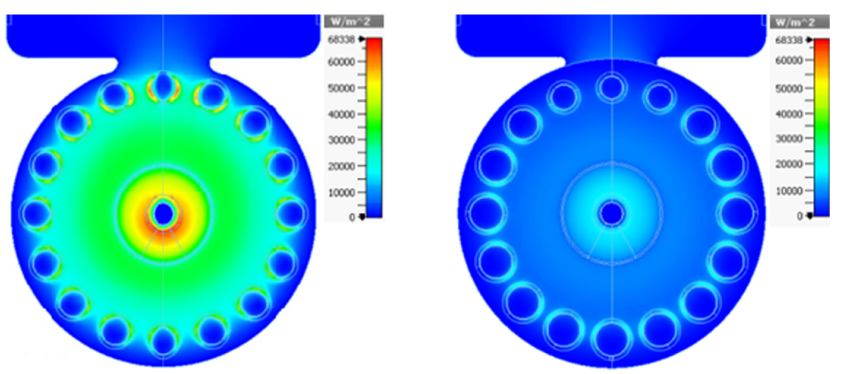

FIG. 4. Variation of $S_{c}$ in the nominal output cell (left) where the power is fed in the tested direction and the nominal input cell (right).

(Fig. 2 left). As a result, the coupling cells provide the same acceleration as the regular cells (Fig. 2 right).

However, since the structure was tested in the reversed direction, which caused significant differences in the field distributions during the test compared to the design. The longitudinal profiles are shown in Fig. 3, where the profile with the power fed into the nominal input is shown in solid line and that with the power fed into nominal output in dashed line. With power fed into the nominal input port, the field profiles are nearly flat. With power fed into the nominal output port, which has a lower group velocity than at the nominal input port, the field distribution is no longer uniform and the maximum field is in the input cell and decreases rapidly toward the end. In addition, the peak $S_{c}$ on the nose of the input cell is about $30 \%$ higher than that in the coupling holes whereas these values are roughly the same when the power is fed in nominal direction. Figure 4 shows the $S_{c}$ variation on the face of the coupling cell with $1 \mathrm{~W}$ input to that cell.

The most important parameters of the BTW accelerating structure in the normal, forward direction, are summarized in Table I.

TABLE I. Main parameters of the accelerating structure.

\begin{tabular}{lc}
\hline \hline Parameters (unit) & Values \\
\hline Frequency (GHz) & 2.9985 \\
Phase advance per sell (rad) & $5 \pi / 6$ \\
Phase velocity (\% of c) & 0.38 \\
Pulse width $(\mu \mathrm{s})$ & 2.5 \\
Average accelerating gradient $(\mathrm{MV} / \mathrm{m})$ & 50 \\
Number of cells & 12 \\
Structure length (mm) & 189.9 \\
Quality factor (first/last cell) & $6954 / 7415$ \\
Normalized shunt impedance $(\mathrm{M} \Omega / \mathrm{m})$ & $51.5 / 54.6$ \\
Filling time (ns) & 224 \\
Group velocity (first/last cell) $(\% \mathrm{c})$ & $0.39 / 0.21$ \\
Peak input power $(\mathrm{MW})$ & 20.6 \\
Max $S_{c} / E_{a}^{2}(\mathrm{~A} / \mathrm{V})$ & $3.1 \times 10^{-4}$ \\
Max $E_{s}(\mathrm{MV} / \mathrm{m})$ & 219 \\
\hline \hline
\end{tabular}




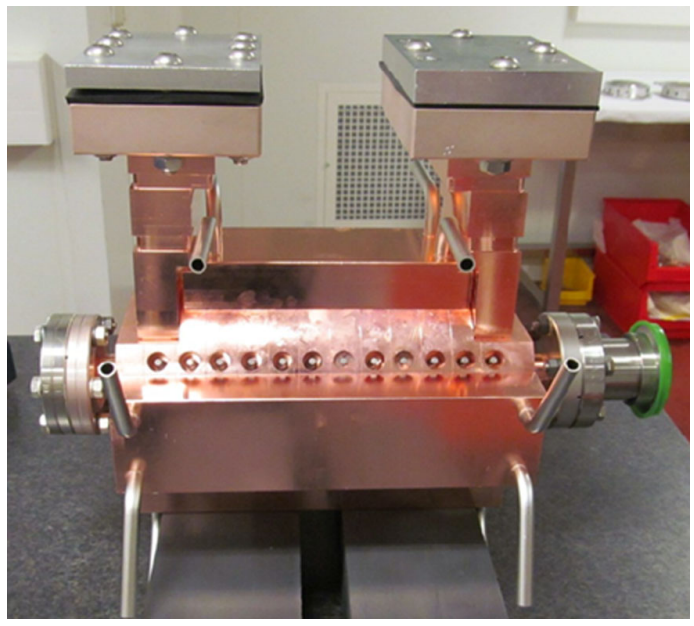

FIG. 5. Picture of the BTW prototype.

\section{MECHANICAL DESIGN AND FABRICATION}

The mechanical design and fabrication methods used for this structure are based on those used for CLIC high gradient $\mathrm{X}$-band accelerating structures $[3,11]$. For example, the cells were fabricated using micron-precision turning and milling followed by bonding and brazing heat cycles with partial hydrogen pressure for assembly. However the mechanical design takes into account many subtleties given by the differing size, rf design, and requirements of this accelerating structure. For example, the slots in the input and output couplers were made part of the end-cells to avoid brazing in the area critical for the matching. Furthermore, the choice of wall thickness and slot sizes results from a compromise between the optimal rf performance and compactness requirements on the one hand, and the minimum wall cross section on the other; the latter has to be large enough to provide structural strength to the cell and to efficiently cool thermal loads in the nose area.

The micron-precision machined disks were manufactured by a commercial supplier [16]. After delivery to CERN, the disks were visually inspected and critical dimensions were measured. For assembly the disks were axially aligned using a precise V-shaped support. The disks were then bonded in partial hydrogen atmosphere, with a maximum temperature of the heat cycle of $1040^{\circ} \mathrm{C}$. Azimuthal alignment was established by using the tuning holes and was checked via fiducial grooves on the external surfaces of the cells. Waveguide transitions, cooling plates and vacuum flanges were brazed together, and then joined to the accelerating structure via a later brazing step. Tuning pins were also brazed to the structure allowing push-pull tuning. An overall view of the completed BTW prototype is shown in Fig. 5.

\section{IV. rf PERFORMANCE AND TUNING}

All 12 cells (10 regular and 2 coupling cells) of the structure were tuned using the bead-pull technique to minimize the standing wave pattern and to provide the desired phase advance ( $150 \pm 1.5^{\circ}$ for regular cells) at the operating frequency of $2.9985 \mathrm{GHz}$ under vacuum at a temperature of $32^{\circ} \mathrm{C}$. The frequency of the output coupling cell was increased by $2.2 \mathrm{MHz}$, the frequency of the 10 regular cells were increased by 0.1 to $0.8 \mathrm{MHz}$ (average of $0.3 \mathrm{MHz}$ ) and the frequency of the input coupling cell was decreased by $0.6 \mathrm{MHz}$. Figure 6 shows the electric field pattern along the structure as well as the measured S-parameters.

\section{HIGH POWER TEST SETUP}

The high-power test of the first BTW prototype was performed in the Sbox test area at CERN, which was formerly part of CTF2 and is now used for high-power S-band and X-band tests [17,18]. The structure was powered by a 43 MW S-band klystron [19] connected to the structure via SF6-filled WR-284 wave guides followed by a window. The klystron was protected against reflected power by a high-power circulator. An ion pump was connected to the prototype through an rf pumping port to maintain a vacuum measured at the pump of below $10^{-8}$ mbar. A picture of the structure and experimental setup installed in the CTF2 bunker is shown in Fig. 7.
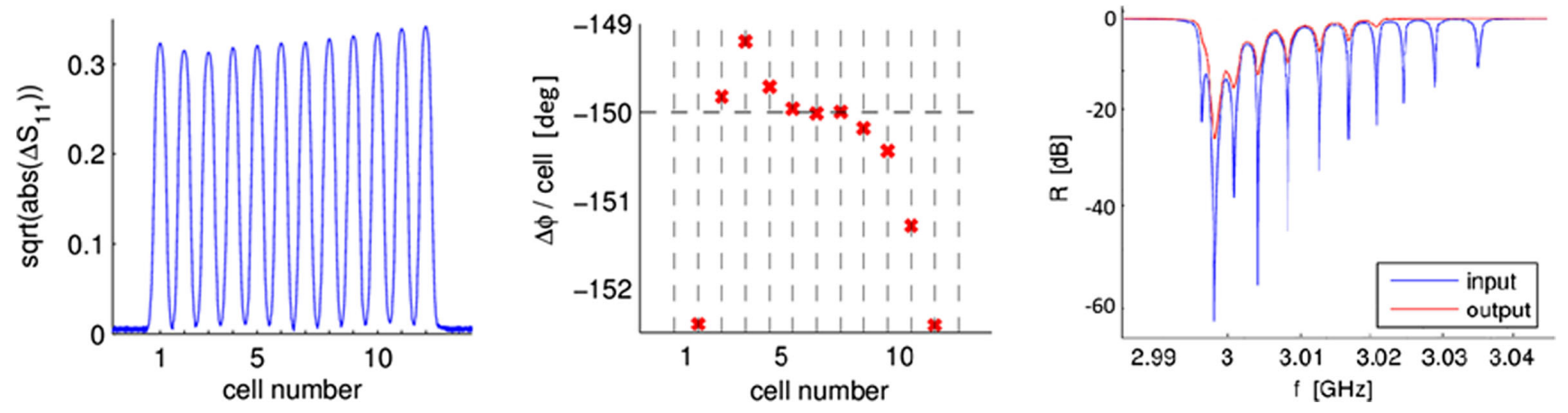

FIG. 6. Measured electric field pattern in magnitude (left) and phase (middle) and the input and output S11 values (right) of the tuned structure in a dry nitrogen atmosphere at $23^{\circ} \mathrm{C}$. 


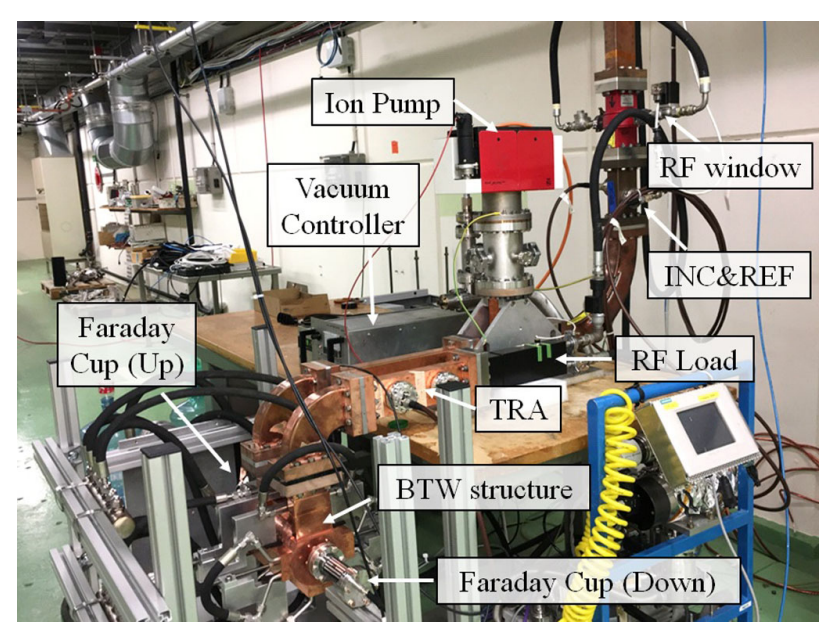

FIG. 7. Picture of structure and subsystems in the Sbox test area at CERN.

The layout, instrumentation, control, BD detection, $\mathrm{BD}$ recovery and conditioning strategy was based on the hardware, electronics, software and algorithms developed and used in the X-band, high-gradient test stands, Xboxes-1 to $3[20,21]$. Nearly twenty CLIC prototype accelerating structures have been successfully conditioned and operated for many thousands of hours at gradients around $100 \mathrm{MV} / \mathrm{m}$ [22-24]. Using the X-band testing methodology for the test of this structure exploits the accumulated experience and also allows the most direct comparison of results.

The acquisition and control system is based on National Instrument electronics [25]. The system allows the user to control the power level, modulate the phase waveform and automatize the conditioning of the structure. A PXI realtime system equipped with FPGA-based digitizers performed the data acquisition of the rf signals, the detection and data storage of BD events. The operation of the test stand is controlled and interfaced by a simplified version of the Xbox-2 LabVIEW software [21].

Directional couplers before and after the structure were used to measure the incident, reflected and transmitted rf signals. The amplitude and phase of these $3 \mathrm{GHz}$ signals were down-mixed to $62.5 \mathrm{MHz}$ and digitized in $250 \mathrm{Msps}$ analog-to-digital converters (ADCs). The incident, reflected and transmitted power signals were calibrated and fitted parameters were used in the Labview code to convert the $\mathrm{mV}$ signals read by the PXI crate into MW rf power values. Additional calibrations were performed periodically during the test and the results were incorporated into the analysis code. On-axis Faraday cups upstream and downstream of the structure measured emitted dark current and BD current bursts from the structure.

The temperature of the structure was stabilized using a stand-alone chiller unit that maintained the input cooling water temperature at $32{ }^{\circ} \mathrm{C}$. Additionally a sensor was used to measure the surface temperature of the structure.
A detailed description of the experimental stand is presented in [26,27].

\section{HIGH-GRADIENT TESTING RESULTS AND ANALYSIS}

Throughout this section, field values were taken into account the reversed installation of the structure. Some values may differ from those found in past publications before the reversed installation was uncovered $[26,28]$. The structure was conditioned using the same computer-controlled algorithm as that used in the Xboxes [18,29]. A number of measurements which are routinely carried out in the CLIC high-gradient tests were repeated on this structure.

The essence of the conditioning algorithm is that the input power is increased by a user-defined power step if no BDs occur in a fixed number of pulses. In addition, the breakdown rate (BDR) is regulated to stay below a threshold value, typically about $3 \times 10^{-5}$ per pulse, by setting of the power step to zero if the BDR threshold is exceeded. The structure was initially operated at the pulse length of $350 \mathrm{~ns}$, conditioned to a gradient in the first cell of $70 \mathrm{MV} / \mathrm{m}$ and then the pulse length was progressively increased to $1.6 \mu \mathrm{s}$. The pulse repetition rate was $25 \mathrm{~Hz}$ at the beginning of the testing and $50 \mathrm{~Hz}$ after 360 million pulses due to a system upgrade. A conditioning history with gradient and accumulated BDs plotted as a function of the number of rf pulses is shown in Fig. 8.

Overall, the structure conditioned very similarly to CLIC structures with an initial period during which the gradient increased, followed by a period during which the pulse length was increased at constant gradient and finally a period when BDR decreased during operation at a fixed gradient and pulse length. The structure achieved a maximum accelerating gradient in the first cell above $80 \mathrm{MV} / \mathrm{m}$ with $1.6 \mu$ s pulses. The test was completed due to a time

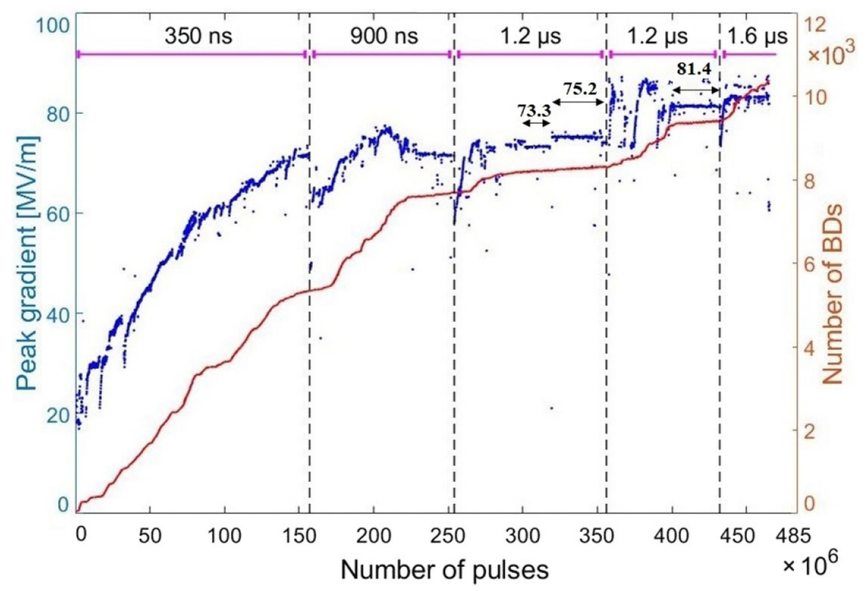

FIG. 8. Conditioning history of the BTW accelerating structure. The plot shows the accelerating gradient in the first cell (blue) and accumulated number of BDs (red) versus the number of pulses, with pulse lengths indicated at the top. 
limit. The BDR decrease can be seen during operation with $1.2 \mu$ s pulses by observing that the curve of the accumulated BDs flattens. More detailed comparisons with CLIC structures are presented in Sec. XI.

A standard measurement for high-gradient structures is the dependence of BDR on gradient [4]. This was measured after the initial conditioning period followed by an extended period of running with constant conditions with $1.2 \mu$ s pulse length in the region from 310 to 350 and from 400 to 430 millions pulses as indicated in Fig. 8.

A plot of BDR as a function of peak surface electric field is shown in Fig. 9. This data analysis has been used to improve the structure performance in its conditioning state. In such case, the rate varies with time and has strong dependence on acceleration gradient. The data has been fit with a power law as presented in Eq. (2). The resulting exponent is significantly lower than the typical power index value of 30 observed in other high gradient structures at 12 and $30 \mathrm{GHz}[5,30-32]$ :

$$
\mathrm{BDR} \propto E_{s}^{10 \pm 3}
$$

Another important measure of the high-gradient behavior of the structure is the distribution of the BDs along the length of the structure. The longitudinal position of a BD can be determined from the relative timing of the rising edge of reflected power and the falling edge of the transmitted power for the BD pulse, including the filling time of the structure to ensure the same time origin since both signals are extracted from two different directional couplers. The distribution of $\mathrm{BD}$ positions for the entire run of the structure obtained using this so-called edge method [33-35] is shown in Fig. 10. BDs occurred predominantly toward the input of the structure. This distribution is consistent with the rapidly increasing field profile toward the input cell, as shown in Fig. 3. The decrease in BDR along the structure is however less rapid than the dependence of the structure as a whole, as described in Eq. (2). This is generally consistent with other structure tests $[3,4]$.

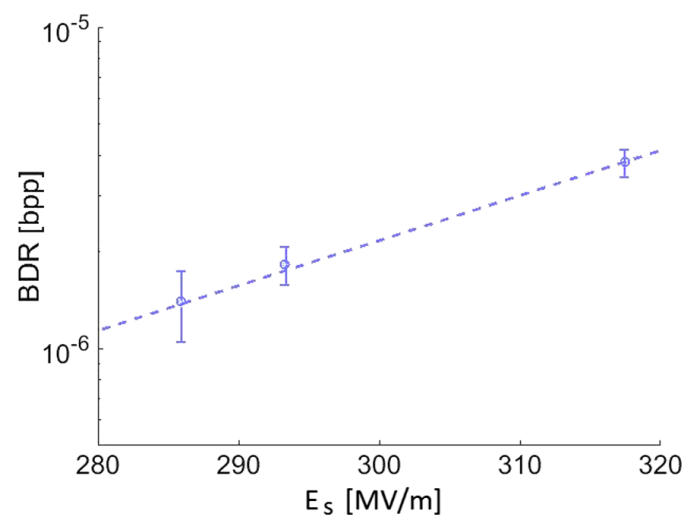

FIG. 9. Measurement of BDR as a function of peak surface electric field. The dashed line shows the power law fit $E_{s}^{10}$.

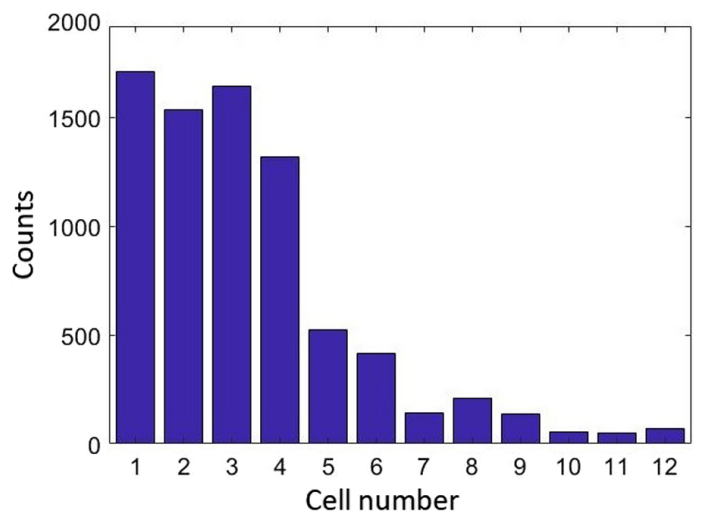

FIG. 10. BDs localization obtained by edge method.

Pulse heating is also one of the driving mechanism of the BDs. However, the maximum value of pulsed surface heating reached during the test was $32^{\circ} \mathrm{C}$, occurring in the uppermost coupling hole in the input coupler, as shown in Fig. 1. This value is below the $40^{\circ} \mathrm{C}$ occurring in, for example, CLIC structure tests [36-38]. In these tests the pulsed heating was shown not to play a role, suggesting that pulsed surface heating does not play a role in this test either.

\section{INVESTIGATION OF LOCATIONS OF BREAKDOWNS AND LIMITING VALUES $S_{c}$}

$S_{c}$, which is a measure of local power flow [5], is an empirical measure of high gradient capability and has been used for example to successfully increase the gradient in successive generations of CLIC accelerating structure designs. In the accelerating structures tested so far, the maximum value of $S_{c}$ occurs in a region of high electric field, and tests have resulted in consistent limiting values $[39,40]$. However in the present structure, $S_{c}$ has local maxima both on the noses, where the electric field is high, and on the coupling holes, where the magnetic field is high. In the nominal powering direction the maximum values of $S_{c}$ are approximately the same on the nose and on the coupling hole whereas in the reversed (tested) direction the maximum value on the nose is approximately $30 \%$ higher than on the coupling holes of the input cell. The ratio varies along the structure due to different coupling holes radii. This can be seen in Fig. 4. A test of the structure in the nominal direction has the potential to address the important question if BDs are equally likely to occur, for the same $S_{c}$, on the coupling irises where the fields are primarily magnetic as on the noses where the fields are primarily electric. This has both practical consequences for further design optimization, but also for a deepened understanding of the BD process. Because $S_{c}$ is higher in the reversed direction this question could not be addressed directly. Nonetheless the BD data, specifically the characteristics of the reflected signals, was analysed with the objective to see if two populations could be identified which might then be used to distinguish nose BDs from coupling hole BDs. 

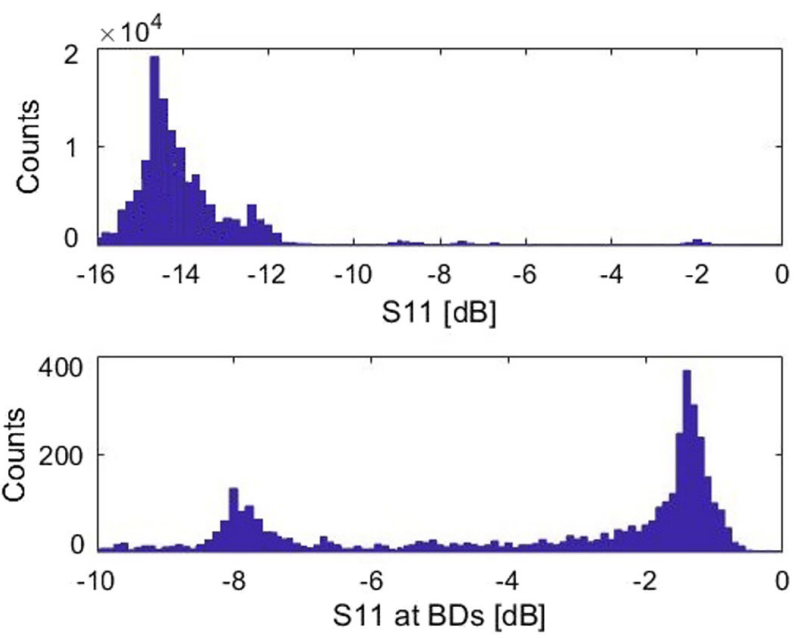

FIG. 11. $S_{11}$ histograms during normal pulses (top) and BD events (bottom) as a function of input reflection $S_{11}$.

In fact, the reflected signals during BD show two distinct peaks in the amplitude population during the runs from $900 \mathrm{~ns}$ to $1.6 \mu \mathrm{s}$ : one with a large reflection coefficient and one with a relatively small one. This can be seen in Fig. 11. Test with a short pulse length is not considered due to the uncertainty in the shape of the reflected signal. Actual pulse shapes of two types of BDs appearing in the two peaks are shown in Fig. 12. The attenuation of the structure was not included in this distribution since most of the BDs are at the front of the structure. Including attenuation would result in only a small correction. The observation of this twinpeaked distribution lead to further investigation if the origin could be correlated with the BD location within the cell. The hypothesis is that BDs on the nose should cause a strong cell detuning, resulting in a large $S_{11}$ while BDs on one of the 16 coupling holes results in a small change in the coupling and consequently a relatively small $S_{11}$.

HFSS simulations were performed to investigate the validity of this hypothesis by simulating a BD with a short circuiting conductor in the two different positions in the cavity. Details of this simulation are described in [13]. According to the simulating, the reflection from the noses is
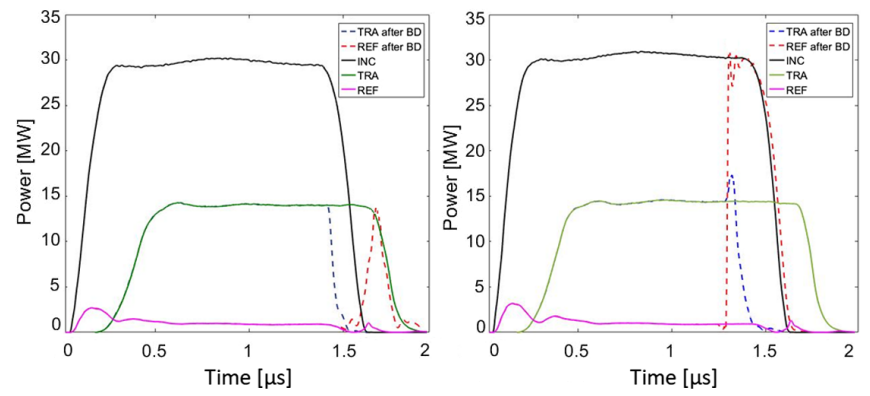

FIG. 12. A nominal incident (INC), reflected (REF) and transmitted (TRA) rf signals in the structure with comparison to a pulse after BD event with different reflection coefficient.

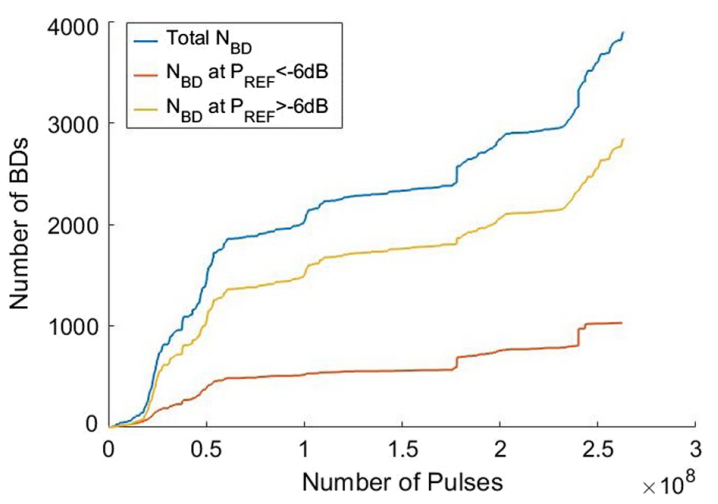

FIG. 13. Numbers of BDs with reflection coefficient $P_{\mathrm{REF}}$ during the conditioning of the BTW structure.

in the range from 0 to $-5 \mathrm{~dB}$, while that on the coupling holes from -6 to $-15 \mathrm{~dB}$. The results of the simulation are consistent with the hypothesis described above, that the $S_{11}$ is much higher when the BD happens on the rf gap, rather than on coupling holes. By taking an input reflection coefficient $P_{\mathrm{REF}}$ of $-6 \mathrm{~dB}$ as the cut-off value for separating the two $\mathrm{BD}$ populations, the number of $\mathrm{BDs}$ occurred on the nose region is greater than on a coupling hole region. This can be seen in Fig. 13. The analysis of endoscope images gives additional information on the question of BD location within a cell as described in Chapter X.

\section{DARK CURRENT STUDIES}

Another important phenomenon which occurs in structure operating at a high-gradient is electron field emission and resulting dark current. Dark current can provide insight into the state of the structure surface and has operational consequences like causing background in current and beam position monitors, radiation if dark currents are accelerated over longer distances and in the extreme case, beam loading of fields. This structure is unique as it has a very high surface fields, in excess of $200 \mathrm{MV} / \mathrm{m}$, and a phase velocity that is much less than $c$. The low phase velocity means that field emitted electrons are more easily captured in an rf bucket, but also that they can exceed the speed of the bucket. Consequently the dynamics of the transported current is much more complicated than in a structure with speed of light phase velocity. An attempt to quantify this effect was performed [13]. To the authors knowledge, this is the first structure which has both high gradient as well as low group velocity.

A plot of dark current measured in the Faraday cups as function input rf power is shown in Fig. 14. The data is also shown in a standard Fowler-Nordheim plot form [41]. The maximum surface electric field in the first cell is used for electric field in this analysis. In addition to the Faraday cup measurement of dark current, radiation outside the structure measured by X-ray photons monitor PAXCT401 can be also used for a Fowler-Nordheim type analysis. This is because 

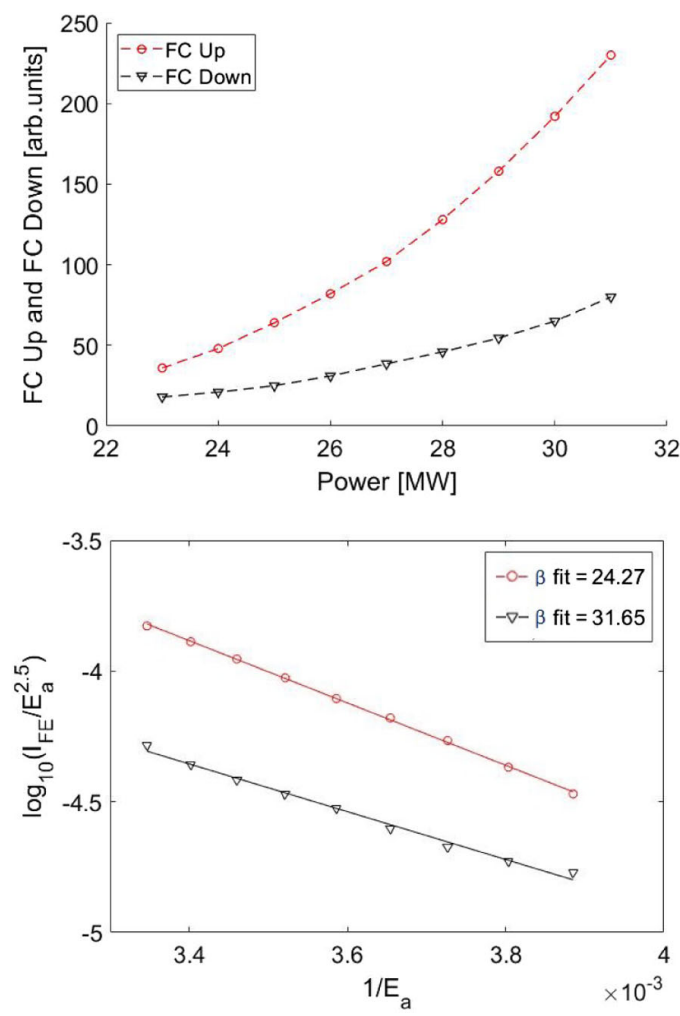

FIG. 14. Dependence of faraday cups signals on power during conditioning (top) and the fit to the Fowler-Nordheim formula (bottom).

under high fields, electrons that are field emitted from the cavity surface are then accelerated by the fields in the cavity volume and finally collide with the walls, producing electron and photon radiation outside the structure (and neutrons when the energy is above around $10 \mathrm{MeV}$ ).

Consequently one can expect a proportionality between dark current measured in Faraday cups and the radiation level outside the structure, which is consistent with measurement as shown in Fig. 15. Figure 16 shows the radiation

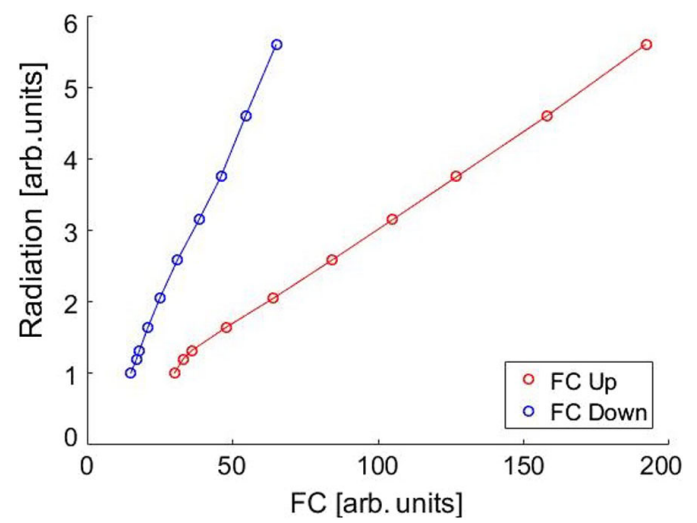

FIG. 15. Dependence of radiation level vs. upstream and downstream faraday cups signals at a pulse length of $1.2 \mu \mathrm{s}$ and an accelerating gradient of $70 \mathrm{MV} / \mathrm{m}$.
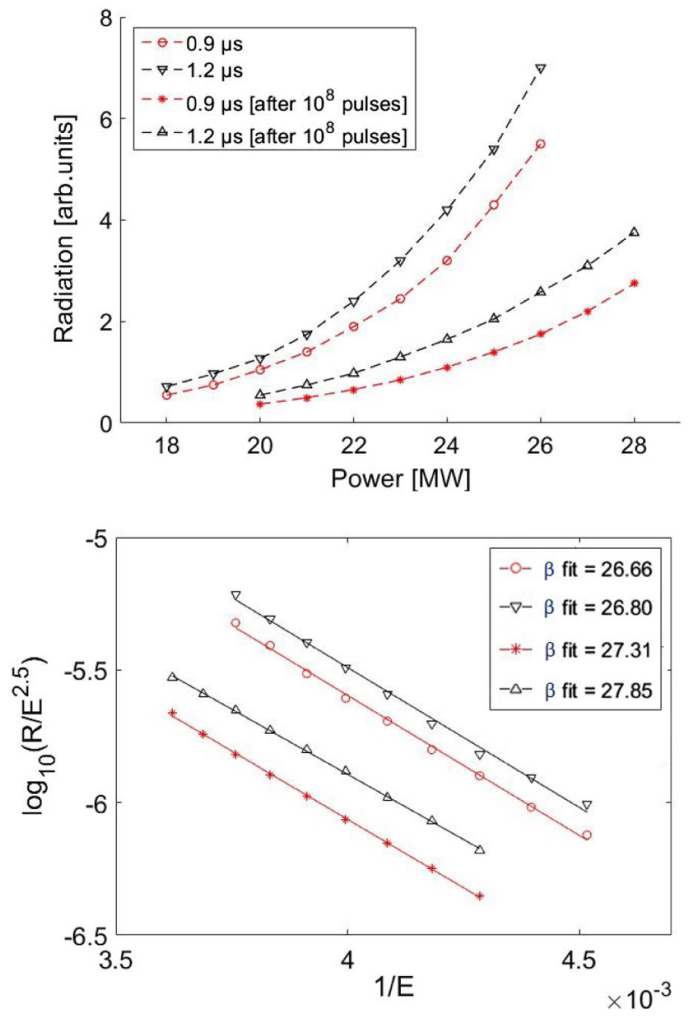

FIG. 16. Dependence of radiation level on power during conditioning (top) and plot in Fowler-Nordheim axes adapted to measured radiation (bottom).

level from the structure as a function of the pulse length and rf power. For this BTW structure, the field enhancement factor $\beta$ is around 27 as shown in the bottom plots of Fig. 14 and 16. It is important to note that enhancement factor stays practically constant during the different stages of the conditioning. The field enhancement value is comparable to the values of $\mathrm{X}$-band accelerating structures tested at CERN [42]. Overall the radiation at a given field level decreases with conditioning time. This is consistent with other experiments [43] and an explanation is given in [44].

\section{POST-TEST rf MEASUREMENT}

After high power testing, the low power rf measurements described in Sec. IV were repeated in order to determine if rf properties changed. The measurement of the complex scattering parameters $S_{11}$ and $S_{22}$, corresponding to the input and output rf ports, show that the magnitude of $S_{11}$ changed from -60.2 to $-19.6 \mathrm{~dB}$ and $S_{22}$ from -24.3 to $-15 \mathrm{~dB}$ at the central frequency $2.9985 \mathrm{GHz}$ (see Fig. 17).

These changes are minor enough that the accelerating structure could be reused without retuning. Still, the structure was tuned again to a state very similar to the one before high power testing by compensating the changes of the few cells at the beginning and end of the structure. The reason for the detuning has not been determined yet however the leading explanation is that cells at the input 

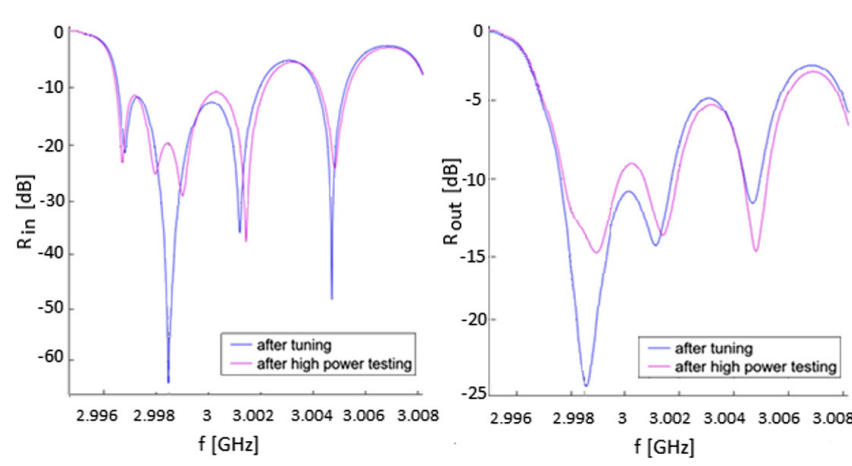

FIG. 17. A comparison of input (left) and output (right) reflection before and after high power testing.

and output of the structure were deformed when forces are applied through the input and output waveguides during installation. These forces may be rather large when for example the rf/vacuum flanges are being bolted together. Future structures will be mechanically reinforced in the area of the input and output waveguide.

\section{BORESCOPE INSPECTION}

After the low power rf measurements were completed, accessible surfaces of the structure were inspected by a borescope. The primary objective of this examination was to determine the location of the BDs to cross check against the longitudinal BDs position distribution obtained from the $\mathrm{rf}$ measurements and to see the relative occurrence of BDs on the nose and on the coupling holes as described in Sec. VII. A selection of images are shown in Fig. 18.

Pictures from the borescope show that most of the BDs occurred on the noses of the cell of the input power side of the structure. The fact that the BD craters are primarily in the input cells confirm the reliability of the data analysis based on the difference in time of the rf signals. However there is evidence of a small amount of BDs on the coupling holes as can be seen in Figs. 18. This confirms the explanation for the double peaked population of BD reflection coefficients presented in Sec. VII. Further investigation of the origin of this effect awaits retest of the structure oriented in the correct direction. The test will be made during the next run of Sbox at CERN at 2020.

\section{CONDITIONING COMPARISON TO CLIC PROTOTYPES}

One of the objectives for designing and testing this structure is to demonstrate that low velocity particles can be accelerated with high gradients, which is required for proton acceleration. The tests also provide data that can be directly compared to that from the speed-of-light CLIC $\mathrm{X}$-band structures in order to determine the range of validity of gradient-limiting parameters.

The major differences to the CLIC structures include lower frequency, $S$ rather than X-band, magnetic coupling
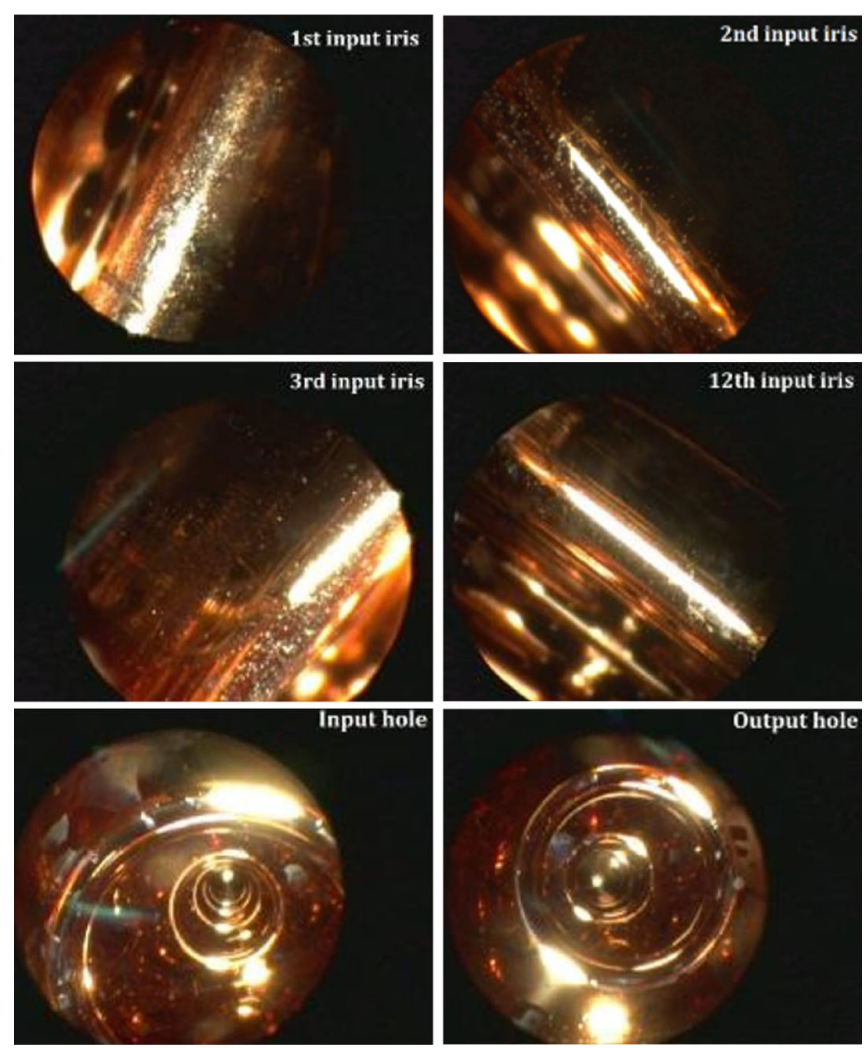

FIG. 18. Borescopy images from the structure after conditioning: the iris of the first, second (top), third, and twelfth (middle) cells, one of the coupling holes of input and output cells (bottom).

rather than electric, $S_{c}$ local maxima in both high electric and magnetic field regions, backward traveling wave rather than forward traveling and standing wave and a low, $v / c=0.38$, phase velocity rather than speed of light. Both confirmation and deviation from expected values contributes to an increased understanding of high-gradient limits and the physical processes behind BD.

Standardized high-gradient measurements were used to make the comparison between this structure and four reference X-band CLIC structures tested at CERN $[29,42]$. These structures were chosen because they were tested under similar conditioning algorithms. Conditioning curves of this structure along with the four reference structures are plotted in terms of unscaled (that is not scaled for pulse length or BDR) surface electric field and modified Poynting vector in Fig. 19. The curves have roughly the same shape and show that the structures behaved similarly. There are many differences in the details, but these are due to operational issues with the test stands themselves. Most strikingly, the absolute value of peak surface electric field was significantly higher in this structure than in the reference CLIC structures.

The same data were then replotted with accelerating gradient and $S_{c}^{1 / 2}$ scaled for pulse length and BDR using the scaling law: BDR $\propto E_{a}^{30} t^{5}$ [45]. This scaling has been observed in previous experiments. Plotted in this way in 
Fig. 20 the processing history of the X-band the BTW Sband structures look similar. However according to the result shown in Fig. 9, the power law scaling measured for the BTW structure is $E_{a}^{10}$. Using this parameter, the scaled

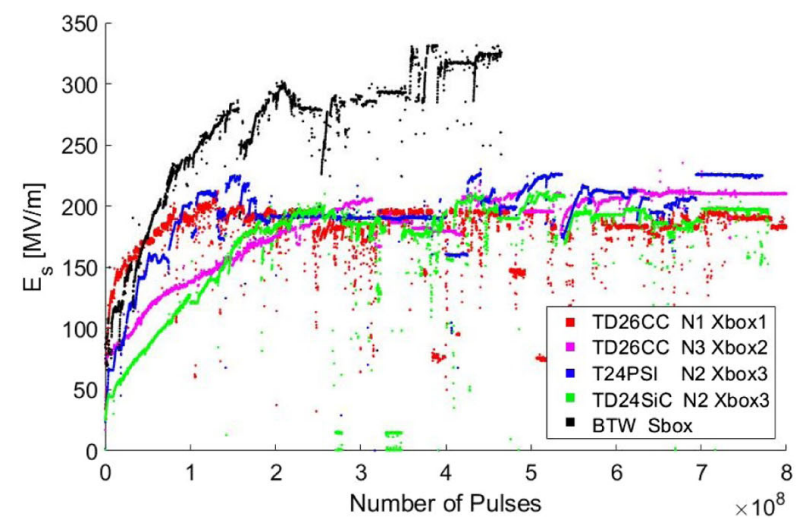

electric field and the scaled modified Poynting vector $S_{c}$ are shown in Fig. 21. Using this scaling the peak electric field again appears significantly higher in this structure than in the CLIC reference structures.

FIG. 19. Comparison of the peak surface electric field $E_{s}$ (left) and the peak modified Poynting vector $S_{c}$ (right) with respect to the number of pulses for: TD26CC N1 (Xbox-1), TD26CC N3 (Xbox-2), T24PSI N2 (Xbox-3), T24SiC N2 (Xbox-3), and BTW N1 (Sbox).
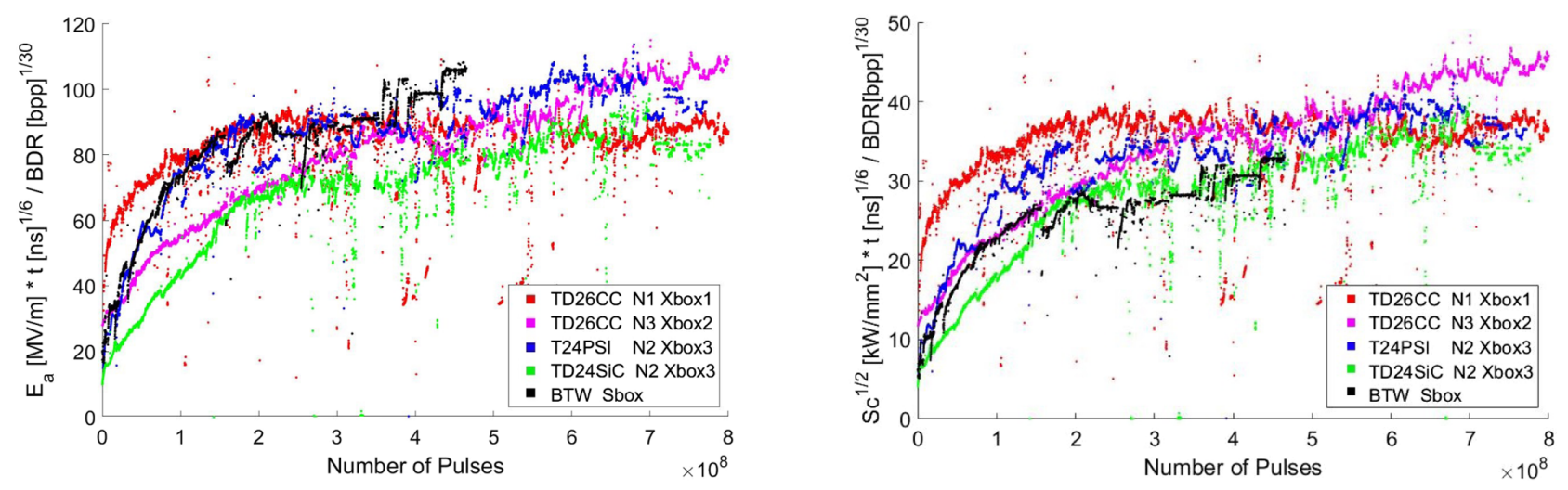

FIG. 20. Comparison of the scaled accelerating gradient (left) of the first cell and the scaled peak modified Poynting vector $S_{c}$ (right) with respect to the number of pulses for: TD26CC N1 (Xbox-1), TD26CC N3 (Xbox-2), T24PSI N2 (Xbox-3), T24SiC N2 (Xbox-3), and BTW N1 (Sbox).
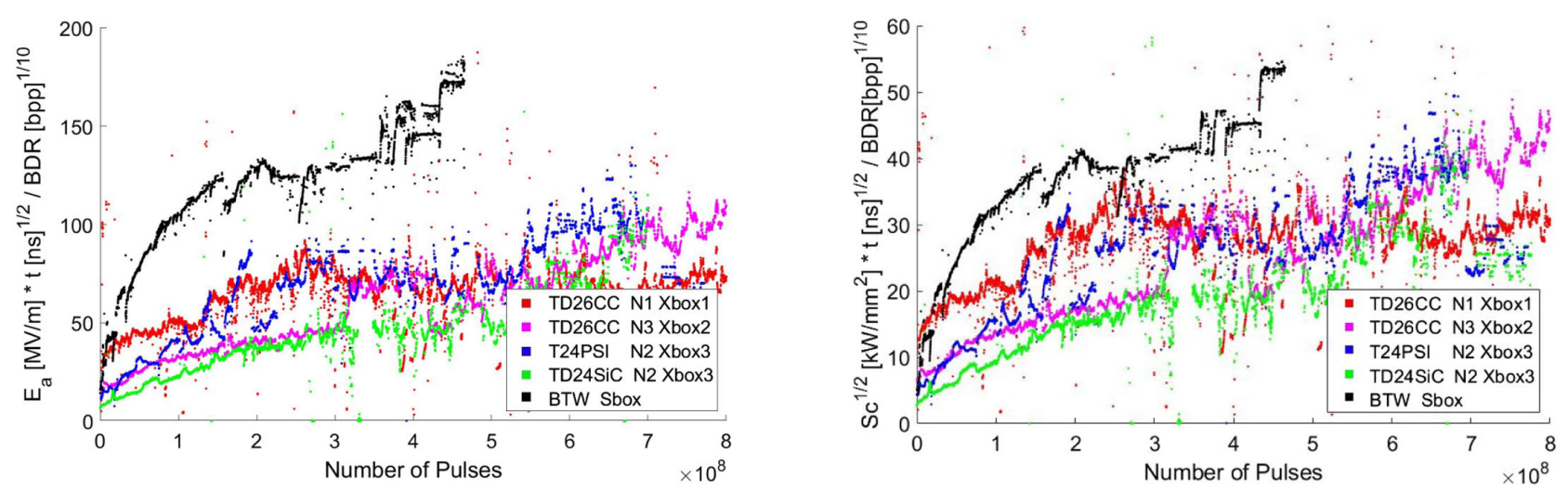

FIG. 21. Comparison of the scaled accelerating gradient (left) of the first cell and the scaled peak modified Poynting vector $S_{c}$ (right) assuming BDR $\propto E_{a}^{10} t^{5}$ with respect to the number of pulses for: TD26CC N1 (Xbox-1), TD26CC N3 (Xbox-2), T24PSI N2 (Xbox-3), T24SiC N2 (Xbox-3), and BTW N1 (Sbox). 
TABLE II. Main operational parameters during the conditioning the accelerating structure at CERN.

\begin{tabular}{lcccccc}
\hline \hline Structure & $f, \mathrm{GHz}$ & $E_{a}, \mathrm{MV} / \mathrm{m}$ & $E_{s}, \mathrm{MV} / \mathrm{m}$ & $S_{c}, \mathrm{MW} / \mathrm{mm}^{2}$ & $t_{p}, \mathrm{~ns}$ & $\mathrm{BDR}, \mathrm{bpp}$ \\
\hline BTW N1 & 3 & 82.6 & 325 & 2.1 & 1600 & $1.9 \mathrm{E}-05$ \\
TD26CC N1 & 12 & 100 & 200 & 3.4 & 200 & $7.5 \mathrm{E}-06$ \\
TD26CC N3 & 12 & 109 & 218 & 5.0 & 200 & $4.6 \mathrm{E}-06$ \\
T24PSI N2 & 12 & 113 & 226 & 4.8 & 200 & $7.5 \mathrm{E}-07$ \\
T24SiC N2 & 12 & 96.5 & 197 & 3.2 & 200 & $1.0 \mathrm{E}-06$ \\
\hline \hline
\end{tabular}

To complete the comparison, a selection of high-gradient parameters achieved after conditioning are summarized in Table II. A detailed description of the total analysis of the BTW structure is presented in [27].

\section{CONCLUSIONS}

A low phase velocity S-band structure has been designed, fabricated and high-power tested. Although the structure was installed backwards, important results were obtained. Firstly, the structure has achieved a local (first few cells) accelerating gradient in excess of $80 \mathrm{MV} / \mathrm{m}$. This shows the potential range of operation for such a structure. This primarily confirms the possibility of using such a low phase velocity structure for the all-linac solution of the TULIP project, which would accelerate proton beams.

At this gradient and corresponding pulse length, the structure was operated with peak surface field values higher than the limiting values in the speed-of-light phase velocity CLIC X-band structures, which have a similar maximum $S_{c}$ but higher acceleration gradient. The conditioning behavior was similar to the CLIC structures but the BDR rate had a much weaker dependence on gradient. Consequently, highgradient technology can be applied in a new domain, in particular in the acceleration of subrelativistic particles.

Two BD locations in a cell was speculated from the two-peaked distribution of BDs analysed with edge method. It is consistent to the artificially inserted short circuits in HFSS simulation, one at the nose and the other at the coupling holes.

A high priority is to test the structure with the power flow in the correct direction. This is important to establish the achievable gradient over an entire structure. The existing structure, and an identical structure fabricated at the same time, will both be tested.

Finally this exercise is an intriguing example of how development in technology for high-energy physics can make an impact also in other fields, such as radiation therapy. The test has shown that the high gradient technology has the potential to provide significant advantages for hadrontherapy linacs.

\section{ACKNOWLEDGMENTS}

The authors would like to thank the rf CLIC group at CERN for their help and continued support. This project has received funding from the European Union's
Horizon 2020 research and innovation programme under the Marie Sklodowska-Curie Grant Agreements Optimization of Medical Accelerators No. 675265 and No. 750871, European Regional Development Funds ERDF FICTS2013-03 and from KE 2638/BE Agreement between IFIC-CSIC and CERN. The authors are also very grateful to the CERN KT group for having funded the accelerating structure prototype construction.

[1] M. Aicheler, P. Burrows, M. Draper, T. Garvey, P. Lebrun, K. Peach, N. Phinney, H. Schmickler, D. Schulte, and N. Toge, A Multi-TeV Linear Collider Based on CLIC Technology: CLIC Conceptual Design Report, CERN Yellow Reports: Monographs (CERN, Geneva, 2012), https://doi.org/10.5170/CERN-2012-007.

[2] J. W. Wang, J. R. Lewandowski, J. W. Van Pelt, C. Yoneda, G. Riddone, G. Dmitry, T. Higo, and T. Takatomi, Fabrication technologies of the high gradient accelerator structures at $100 \mathrm{MV} / \mathrm{m}$ range, in Proceedings of the International Particle Accelerator Conference, Kyoto, Japan (ICR, Kyoto, 2010), Vol. 3, pp. 3819-3821.

[3] A. Grudiev and W. Wuensch, Design of the CLIC main linac accelerating structure for CLIC conceptual design report, in Proceedings of the 25th International Linear Accelerator Conference, LINAC-2010, Tsukuba, Japan (KEK, Tsukuba, Japan, 2010), pp. 211-213.

[4] A. Degiovanni, W. Wuensch, and J. G. Navarro, Comparison of the conditioning of high gradient accelerating structures, Phys. Rev. Accel. Beams 19, 032001 (2016).

[5] A. Grudiev, S. Calatroni, and W. Wuensch, New local field quantity describing the high gradient limit of accelerating structures, Phys. Rev. Accel. Beams 12, 102001 (2009).

[6] ADAM website, https://www.avoplc.com/en-gb/Technology/ Overview-of-the-LIGHT-System.

[7] A. Degiovanni and U. Amaldi, Proton and Carbon Linacs for Hadron Therapy, in Proceeding of LINAC 2014, FRIOB02, Report No. CERN-ACC-2014-362 (Geneva, Switzerland, 2014), pp. 1207-1212.

[8] C. De Martinis, D. Giove, U. Amaldi, P. Berra, K. Crandall, M. Mauri, M. Weiss, R. Zennaro, E. Rosso, B. Szeless, M. Vretenar, M. Masullo, V. Vaccaro, L. Calabretta, and A. Rovelli, Acceleration tests of a $3 \mathrm{GHz}$ proton linear accelerator (LIBO) for hadrontherapy, Nucl. Instrum. Methods Phys. Res., Sect. A 681, 10 (2012).

[9] S. Benedetti, T. Argyropoulos, C. B. Gutirrez, N. C. Lasheras, A. Degiovanni, D. E. Pereira, M. Garlasch, J. G. Navarro, A. Grudiev, G. McMonagle, A. Solodko, 
M. Timmins, R. Wegner, B. Woolley, and W. Wuensch, Fabrication and testing of a novel S-Band backward travelling wave accelerating structure for proton therapy linacs, in Proceeding of LINAC 2016, MOPLR048 (East Lansing, MI, USA, 2017), pp. 237-239, https:// doi.org/10.18429/JACoW-LINAC2016-MOPLR048.

[10] S. Benedetti, A. Grudiev, and A. Latina, High gradient linac for proton therapy, Phys. Rev. Accel. Beams 20, 040101, p. 19 (2017).

[11] A. Degiovanni, High gradient proton linacs for medical applications, Ph.D. thesis, Ecole Polytechnique Federale de Lausanne, 2014.

[12] S. Benedetti, U. Amaldi, A. Degiovanni, A. Grudiev, and W. Wuensch, RF Design of a Novel S-Band Backward Traveling Wave Linac for Proton Therapy, in Proceeding of LINAC 2014 (Geneva, Switzerland, 2014), pp. 992-994, pp. THPP061, http://cds.cern.ch/record/2062620.

[13] S. Benedetti, High-gradient and high-efficiency linear accelerators for hadron therapy, Ph.D. thesis, Ecole Polytechnique Federale de Lausanne, 2018.

[14] U. Amaldi, S. Braccini, and P. Puggioni, High frequency linacs for hadron therapy, Reviews of Accelerator Science and Technology (RAST) (World Scientific, Singapore, 2009), Vol. II, p. 111, https://doi.org/10.1142/ 9789814299350_0006.

[15] A. Degiovanni, U. Amaldi, R. Bonomi, M. Garlasche, A. Garonna, S. Verdu-Andres, and R. Wegner, TERA high gradient test program of $\mathrm{rf}$ cavities for medical linear accelerators, Nucl. Instrum. Methods Phys. Res., Sect. A 657, 55 (2011).

[16] VDL Groep (Van Der Leegte Groep) website, https://www .vdlgroep.com/en.

[17] CLIC Collaboration, CTF3 Design Report No. CERN/PS 2002-008, CTF3 Note 2002-047, LNF-02/008, 2002.

[18] N. Catalan-Lasheras, A. Degiovanni, S. Doebert, W. Farabolini, J. Kovermann, G. McMonagle, S. Rey, I. Syratchev, L. Timeo, W. Wuensch, B. Woolley, and J. Tagg, Experience Operating an X-band High-Power Test Stand at CERN, in Proceeding of IPAC 2014 (2014), pp. 2288-2290, https://cds.cern.ch/record/1742951.

[19] G. Geschonke and A. Ghigo, CTF3 Design Report, CERN, Geneva, CERN-PS-2002-008-RF, CTF3-NOTE-2002-047, LNF-2002-008-IR, 2002.

[20] F. Peauger, A. Hamdi, S. Curt, S. Doebert, G. McMonagle, G. Rossat, K. Schirm, I. Syratchev, L. Timeo, S. Kuzikhov, A. Vikharev, A. Haase, D. Sprehn, and A. Jensen, A 12 GHz rf power source for the clic study, in Proceedings of the 25th International Linear Accelerator Conference, LINAC-2010, Tsukuba, Japan (KEK, Tsukuba, Japan, 2010), pp. 3990-3992.

[21] B. Woolley, High power X-band RF test stand development and high power testing of the CLIC crab cavity, Ph.D. thesis, Lancaster University, 2015.

[22] T. Argyropoulos, N. Catalan-Lasheras, A. Grudiev, G. Mcmonagle, E. Rodriguez-Castro, I. Syrachev, R. Wegner, B. Woolley, W. Wuensch, H. Zha, V. Dolgashev, G. Bowden, A. Haase, T. G. Lucas, M. Volpi, D. EsperantePereira, and R. Rajamki, Design, fabrication, and highgradient testing of an X-band, traveling-wave accelerating structure milled from copper halves, Phys. Rev. Accel. Beams 21, 061001 (2018).

[23] X. Wu, J. Shi, H. Chen, J. Shao, T. Abe, T. Higo, S. Matsumoto, and W. Wuensch, High-gradient breakdown studies of an X-band Compact Linear Collider prototype structure, Phys. Rev. Accel. Beams 20, 052001 (2017).

[24] W. Wuensch, A review of vacuum breakdown in highgradient accelerators, 28th International Symposium on Discharges and Electrical Insulation in Vacuum (ISDEIV) 20, 52001 (2018).

[25] LabVIEW, National Instruments, website, https://www.ni .com/labview/.

[26] A. Vnuchenko, S. Benedetti, C. Blanch Gutierrez, N. Catalan Lasheras, D. Esperante Pereira, A. Faus-Golfe, A. Grudiev, B. Koubek, T. G. Lucas, G. McMonagle, S. Pitman, I. Syratchev, M. Volpi, B. J. Woolley, and W. Wuensch, High gradient performance of an S-band backward traveling wave accelerating structure for medical hadron therapy accelerators, in Proceeding of IPAC 2018, Vancouver, BC, Canada, International Particle Accelerator Conference No. 9 (JACoW Publishing, Geneva, Switzerland, 2018), pp. 491-494, https://doi.org/ 10.18429/JACoW-IPAC2018-MOPML043.

[27] A. Vnuchenko, High-Gradient issues in S-band RF acceleration structure for hadron therapy accelerators and Radio Frequency Quadrupoles, Ph.D. thesis, University of Valencia, 2020, http://roderic.uv.es/handle/10550/ 73181 ?show=full.

[28] S. Benedetti, New Trends in Proton and Carbon Therapy Linacs, in Proceeding of LINAC 2018 (2018), pp. 666-670, https://doi.org/10.18429/JACoW-LINAC2018TH1P03.

[29] A. Degiovanni, S. Doebert, W. Farabolini, A. Grudiev, J. Kovermann, E. Montessinos, I. Syratchev, R. Wegner, W. Wuensch, A. Solodko, and B. Woolley, High-Gradient test results from a CLIC prototype accelerating structure: TD26CC, in Proceeding of IPAC2014 (2014), https:// cds.cern.ch/record/1742280.

[30] W. Wuensch, H. Aksakal, H. Braun, G. Carron, R. Corsini, S. Doebert, R. Fandos, A. Grudiev, E. Jensen, O. Mete, T. Ramsvik, J. A. Rodrguez, J. P. Sladen, I. Syratchev, M. Taborelli, F. Tecker, P. Urschtz, and I. H. Wilson, A high-gradient test of a $30 \mathrm{GHz}$ molybdenum-iris structure, Proceedings of EPAC 2006, Edinburgh, Scotland (2006), p. 801 [Reports No. CERN-AB-2006-035, CLIC-Note680], http://cds.cern.ch/record/971788.

[31] S. Matsumoto, T. Abe, Y. Higashi, T. Higo, and Y. Du, High gradient test at Nextef and high-power long-term operation of devices, Nucl. Instrum. Methods Phys. Res., Sect. A 657, 160 (2011).

[32] S. Verd-Andrs, High-gradient accelerating structure studies and their application in hadrontherapy, $\mathrm{Ph} . \mathrm{D}$. thesis, University of Valencia, 2013.

[33] C. Adolphsen, Normal-conducting rf structure test facilities and results, in Proceedings of the 20th Particle Accelerator Conference, PAC-2003, Portland, OR, 2003 (IEEE, New York, 2003), pp. 668-672.

[34] A. Degiovanni, S. Doebert, W. Farabolini, I. Syratchev, W. Wuensch, J. G. Navarro, J. Tagg, and B. Woolley, 
Diagnostics and analysis techniques for high power $\mathrm{X}$ band accelerating structures, in Proceeding of LINAC 2014 (Geneva, Switzerland, 2014), pp. 490-492, http://cds.cern .ch/record/2025952.

[35] A. Palaia, V. Dolgashev, J. Lewandowski, and S. Weathersby, Diagnostics of RF breakdowns in highgradient accelerating structures, in Proceedings of the 4th International Particle Accelerator Conference, IPAC-2013, Shanghai, China, 2013 (JACoW, Shanghai, China, 2013), pp. 527-529.

[36] J. J. Huopana and S. T. Heikkinen, Thermo-structural analysis of the rf-induced pulsed surface heating of the CLIC accelerating structures, Tech. Report No. CERN-OPEN2007-002, CLIC-Note-702, CERN, Geneva, 2006, https:// cds.cern.ch/record/1012908.

[37] K. N. Sjobaek, Avoiding vacuum arcs in high gradient normal conducting RF structures, Ph.D. thesis, Oslo University, 2016 [Report No. CERN-THESIS-2016-129].

[38] A. Grudiev, RF constraints. Update 2013 for CLIC rebaselining, Presented at the 6th CLIC design meeting, (April 19th 2013).

[39] W. Wuensch, CLIC Accelerating Structure Development, in Proceedings of the 11th European Particle Accelerator Conference, Genoa, 2008 (EPS-AG, Genoa, Italy, 2008), pp. 2922-2926.

[40] S. Doebert et al., High gradient performance of NLC/GLC $\mathrm{X}$-band accelerating structures, in Proceedings of the $21 \mathrm{st}$
Particle Accelerator Conference, Knoxville, TN, 2005 (IEEE, Piscataway, NJ, 2005), ROAC004, pp. 372-374 [Report No. SLAC-PUB-11207].

[41] J. W. Wang and G. A. Loew, Field emission and RF breakdown in high-gradient room-temperature linac structures, Tech. Report, 1997.

[42] R. Zennaro, H. Blumer, M. Bopp, T. Garvey, L. Rivkin, N. Catalan-Lasheras, A. Grudiev, G. McMonagle, A. Solodko, I. Syratchev, R. Wegner, B. Woolley, W. Wuensch, T. G. Lucas, M. Volpi, and T. Argyropoulos, High-power tests of a prototype X-band accelerating structure for CLIC, in Proceeding of IPAC2017, Copengagen, Denmark (2017), pp. 4318-4320 [Report No. CERN-ACC-2017179; CLIC-Note-1134].

[43] A. Korsbäck, L. M. Morales, I. Profatilova, E. R. Castro, W. Wuensch, S. Calatroni, and T. Ahlgren, Vacuum electrical breakdown conditioning study in a parallel plate electrode pulsed DC system, arXiv:1905.03996.

[44] E. Z. Engelberg, A. B. Yashar, Y. Ashkenazy, M. Assaf, and I. Popov, Theory of electric field breakdown nucleation due to mobile dislocations, Phys. Rev. Accel. Beams 22, 083501 (2019).

[45] A. Degiovanni, R. Bonomi, M. Garlasch, S. VerduAndres, R. Wegner, and U. Amaldi, High gradient RF test results of S-band and C-band cavities for medical linear accelerators, Nucl. Instrum. Methods Phys. Res., Sect. A 890, 1 (2018). 